\title{
Magnetic resonance imaging and symptoms in patients with neurosarcoidosis and central diabetes insipidus
}

\author{
Eiichi Kakehi', Seiji Adachi', Yusuke Fukuyasu', Yasuhiro Hashimoto ${ }^{1}$, Shigehisa Sakurai', \\ Akane Hirotani' ${ }^{1}$ Hisanori Danbara ${ }^{2}$, Kaduyo Shimizu' ${ }^{1}$, Ryosuke Fujita', Hiroyuki Teraura ${ }^{3}$, \\ Kazuhiko Kotani ${ }^{3}$, Masami Matsumura ${ }^{3}$
}

${ }^{1}$ Department of General Medicine, Tottori Municipal Hospital, Tottori-City, Tottori, Japan

${ }^{2}$ Department of Internal Medicine, Tottori Municipal Hospital, Tottori-City, Tottori, Japan

${ }^{3}$ Center for Community Medicine, Jichi Medical University, Shimotsuke-City, Tochigi, Japan

\begin{abstract}
Introduction: In the clinical setting, the diagnosis of neurosarcoidosis in patients with central diabetes insipidus (CDI) is typically based both on symptoms (i.e. polydipsia or polyuria) and brain magnetic resonance imaging (MRI) findings (e.g. pituitary abnormality). However, inconsistent changes in the patient's symptoms and brain MRI findings may occur during the clinical course of the disease.

This review was performed to summarise the relationship between symptoms and brain MRI findings in previously reported cases of neurosarcoidosis with CDI.

Material and methods: Case studies of patients diagnosed with neurosarcoidosis with CDI were collected via a PubMed search of studies published through 30 June 2018.

Results: Thirteen eligible studies were reviewed (20 patients; 12 men, 8 women; mean age 33 years). Polydipsia or polyuria was the first symptom in 13 patients. The mean duration from disease onset to diagnosis was 3.4 months. Brain MRIs showed abnormal findings in the hypothalamus and pituitary for 17 patients. Immunosuppressive drugs were used in 17 patients. For 14 patients, MRI findings improved, while symptoms did not.

Conclusion: Patients with both neurosarcoidosis and CDI symptoms often do not improve, despite the fact that brain MRI findings often improve following treatment. More studies involving detailed pathological analyses and longer follow-up periods are necessary. (Endokrynol Pol 2019; 70 (5): 430-437)
\end{abstract}

Key words: neurosarcoidosis; central diabetes insipidus; pituitary; pituitary stalk; prednisolone

\section{Introduction}

Sarcoidosis is a multi-organ, non-caseating, granuloma-forming disorder. Although its aetiology remains unknown, sarcoidosis is thought to be immune-mediated [1]. Nervous system involvement, which can occur in either the central or peripheral nervous system, is observed in $5 \%$ to $15 \%$ of patients with sarcoidosis [2, 3]. When the hypothalamus-pituitary axis is affected in patients with sarcoidosis, central diabetes insipidus (CDI) is generally also present [4]. CDI is rarely observed, occurring in approximately $2 \%$ of patients with neurosarcoidosis, and presents with unique symptoms, such as polydipsia or polyuria [5].

Magnetic resonance imaging (MRI) with gadolinium is the recommended method for the initial assessment of neural pathologies [6]. Brain MRI can be helpful in patients with neurosarcoidosis, although autoimmune inflammatory, infectious, or malignant diseases can display similar MRI findings as neurosarcoidosis [7]. The MRI findings of neurosarcoidosis typically include infundibular involvement, pituitary stalk thickening, or pituitary gland enlargement; additionally, a loss of the hyperintense signal from the posterior pituitary on T1-weighted images is indicative of an infiltrative process [6].

An earlier report suggested that corticosteroid treatment in patients with neurosarcoidosis effectively ameliorated MRI abnormalities but failed to completely subdue CDI without desmopressin supplementation; however, this study included only four cases [8]. The current review of previously published case studies was performed to summarise the relationship between changes in symptoms and brain MRI findings during the clinical course of neurosarcoidosis with CDI. 


\section{Material and methods}

\section{Search strategy}

We conducted a comprehensive literature search of PubMed to identify studies that reported neurosarcoidosis with CDI. A search of the PubMed database was performed using the terms 'neurosarcoidosis' or 'central nervous system sarcoidosis' and 'diabetes insipidus' to identify any articles published through March 2019. Study selection

Original case reports that included the following data were included in the present review: 1) age and sex; 2) first symptoms; 3) duration of polydipsia or polyuria; 4) affected organs (lungs, eyes, or other organs); 5) anterior pituitary function; 6) brain MRI findings (abnormalities or loss of high signal intensity of the posterior lobe of the pituitary); 7) histological findings; 8) treatment; and 9) clinical course (brain MRI findings and CDI improvement). Review articles and articles written in languages other than English were excluded. After we identified 98 articles, we determined that 13 articles (including 20 patients) were eligible.

\section{Results}

Table I provides a summary and Table II provides a detailed list of the characteristics of the 20 patients who were diagnosed with neurosarcoidosis with CDI. The patients included 12 men $[8-12,14,15,17]$ and eight women $[7,10,13,16,18,19]$, with a mean age of 33 years. The first symptom was polyuria in 13 patients [7-9, 11-17] and polydipsia in eight patients $[7-9,11-15]$. The mean duration from disease onset to diagnosis was 3.4 months. Isolated neurosarcoidosis was reported in four patients [7, 8, 10, 12]. Brain MRI showed abnormal findings in the pituitary stalk in 10 patients $[7,8,10-13,16]$, abnormal findings in the pituitary gland in nine patients [8-13, 15], and loss of high intensity of the posterior lobe of the pituitary in nine patients $[8,9,11-13]$. Twelve patients were diagnosed through biopsies of the lymph nodes [8, 10, 14, 16, 17], lungs [8-10], and skin $[13,15]$, while only one patient underwent a brain biopsy [8].

Table III provides a detailed list of the clinical treatments, brain MRI findings, and outcomes of patients with neurosarcoidosis and CDI. Steroid therapy was administered to 17 patients [8-12, 14-19], infliximab was administered to two patients $[17,19]$, cyclophosphamide was administered to one patient [10], and methotrexate was administered to one patient [19]. Desmopressin (1-desamino-8-D-arginine vasopressin) (DDAVP) was used to treat the symptoms of CDI in 19 patients [7-14, 16-19]. Fourteen patients showed improvement in their abnormal brain MRI findings [8-13, 15-17], but no patients showed improvement in the loss of high signal intensity in the posterior lobe of the pituitary. One patient showed spontaneous improvement in CDI symptoms without the use of an immunosuppressive agent [13]. Five patients showed improvement in their anterior pituitary functions, and improvement in the MRI findings were also recognised in these patients $[8,9,11,16]$.

\section{Discussion}

In the current review, complete improvement in CDI symptoms was not observed in most patients diagnosed with neurosarcoidosis with CDI, despite improvements in the abnormal brain MRI findings. These findings suggest that inconsistent changes in symptoms and brain MRI findings occur in most cases during the clinical course of the disease, and reinforce the observation made in the earlier report that included a small number of cases [8]. This result has clinical implications for the use of MRI in the diagnosis of neurosarcoidosis in patients with CDI.

There are several possible reasons for the findings in this review. Once the central nervous system has become involved in sarcoidosis, the pathological changes that occur in the brain may be irreversible. Some studies have reported improvements in the MRI findings for the anterior pituitary after immunosuppressant treatment $[8,9,11,16]$. In particular, the posterior, rather than anterior, pituitary gland may undergo irreversible damage following granuloma formation. Because the distance from the base of the brain to the sella turcica is greater than that to the pituitary, there has been an assumption that sarcoidosis lesions cannot completely invade the anterior pituitary [16].

Patients with sarcoidosis typically display infundibular involvement, pituitary stalk thickening, or pituitary gland enlargement when examined by MRI, and the loss of the hyperintense signal from the posterior pituitary on T1-weighted images can be indicative of an infiltrative process [6]. Infiltration of sarcoidosis into the infundibulum may jeopardise the production and transport of pituitary-stimulating hormones and induce the direct involvement of the pituitary [2]. This infiltration process may be independent of improvements in CDI symptoms.

The primary treatment option for neurosarcoidosis is steroid therapy [20]. Immunosuppressive agents and antimalarials can also be administered in resistant cases or when glucocorticoids are tolerated [20,21]. In the current review, immunosuppressants, especially prednisolone, were used in most of the cases, but CDI did not improve, and the use of DDAVP was continued. One report suggested that the early administration (within one month of onset) of prednisolone was effective for the improvement of CDI symptoms; however, not all patients had posterior pituitary lesions in that report [22]. In the current review, the mean duration from disease onset to diagnosis was $>3$ months, and the lack of early treatment may have contributed to the inconsistent improvements in symptoms relative to the MRI findings. 
Table I. Clinical diagnoses, treatments, and outcomes for patients with neurosarcoidosis and central diabetes insipidus

\begin{tabular}{|c|c|c|c|c|c|}
\hline Diagnosis & & & Treatments & & \\
\hline Age, years & 33 & $(10-77)$ & Immunosuppressant $(\mathrm{n}=20)$ & & \\
\hline Sex, male & 12 & (60) & PSL & 16 & (80) \\
\hline First manifestations $(n=20)$ & & & $\mathrm{mPSL}$ & 2 & (10) \\
\hline Polyuria & 13 & (65) & Hydrocortisone & 2 & (10) \\
\hline Polydipsia & 8 & (40) & Infliximab & 2 & (10) \\
\hline \multirow{2}{*}{ Duration of polydipsia or polyuria (months) } & \multirow{2}{*}{3.4} & \multirow{2}{*}{$(1-12)$} & Cyclophosphamide & 1 & (5) \\
\hline & & & Methotrexate & 1 & (5) \\
\hline Lesion sites $(n=20)$ & & & Endocrine $(n=20)$ & & \\
\hline Lungs & 13 & (65) & DDAVP & 19 & (95) \\
\hline Eyes & 3 & (15) & Thyroid hormone & 3 & (15) \\
\hline Isolated & 4 & (20) & Gonadotropin hormone & 1 & (5) \\
\hline \multicolumn{6}{|l|}{ Anterior pituitary dysfunction $(\mathrm{n}=20)$} \\
\hline PRL & 12 & (60) & Symptoms and MRI findings & & \\
\hline LH & 9 & (45) & Brain MRI abnormality $(n=20)$ & & \\
\hline FSH & 9 & (45) & Improved & 14 & (70) \\
\hline ACTH & 6 & (30) & Not improved & 3 & (15) \\
\hline TSH & 6 & (30) & NR & 3 & (15) \\
\hline $\mathrm{GH}$ & 4 & (20) & Improvement of loss of high signal intensity* $(n=20)$ & & \\
\hline Panhypopituitarism & 3 & (15) & Improved & 0 & (0) \\
\hline Brain MRI $(n=20)$ & & & No change & 4 & (20) \\
\hline Abnormality & 18 & (90) & NR & 16 & (80) \\
\hline Pituitary stalk & 10 & (50) & Symptoms of CDI $(n=20)$ & & \\
\hline Pituitary gland & 9 & (45) & Improved & 1 & (5) \\
\hline Hypothalamus & 5 & $(25)$ & Not improved & 15 & (75) \\
\hline Loss of high signal intensity* & & & NR & 4 & (20) \\
\hline Yes & 9 & (45) & Anterior pituitary dysfunction $(n=16)$ & & \\
\hline No & 1 & (5) & Improved & 4 & (20) \\
\hline NR & 10 & $(50)$ & Not improved & 4 & (20) \\
\hline Histology $(n=20)$ & & & NR & 8 & (40) \\
\hline Brain & 1 & (5) & Other neurological symptoms $(n=14)$ & & \\
\hline Others & 11 & (55) & Improved & 7 & (50) \\
\hline Lymph node & 6 & (30) & Not improved & 3 & (15) \\
\hline TBLB & 3 & (15) & NR & 4 & (20) \\
\hline Skin & 2 & (10) & & & \\
\hline
\end{tabular}

Data are presented as the mean (range) or $\mathrm{n}(\%) ;{ }^{*}$ posterior lobe of the pituitary gland; PRL — prolactin; LH — luteinising hormone; FSH — follicle stimulating hormone; ACTH — adrenocorticotropic hormone; TSH — thyroid-stimulating hormone; GH — growth hormone; MRI — magnetic resonance imaging; NR — not reported; TBLB — transbronchial lung biopsy; PSL — prednisolone; mPSL — methylprednisolone; DDAVP — 1-desamino-8-D-arginine vasopressin; CDI — central diabetes insipidus

Untreated self-limiting cases and cases with atypical neurosarcoidosis have been reported [10, 13, 23]; additionally, several diseases can present with MRI findings that are similar to those observed for neurosarcoidosis [7]. Substantial experience and cautious judgment appear to be necessary for the accurate diagnosis of neurosarcoidosis in patients with CDI, which could also explain the inconsistencies observed between CDI symptoms and MRI findings.

The current review has several limitations. The number of reports included in the review was relatively small. Because brain biopsies were very rare (only one case in this review), most patients were clinically but not pathologically diagnosed. None of the reviewed studies 


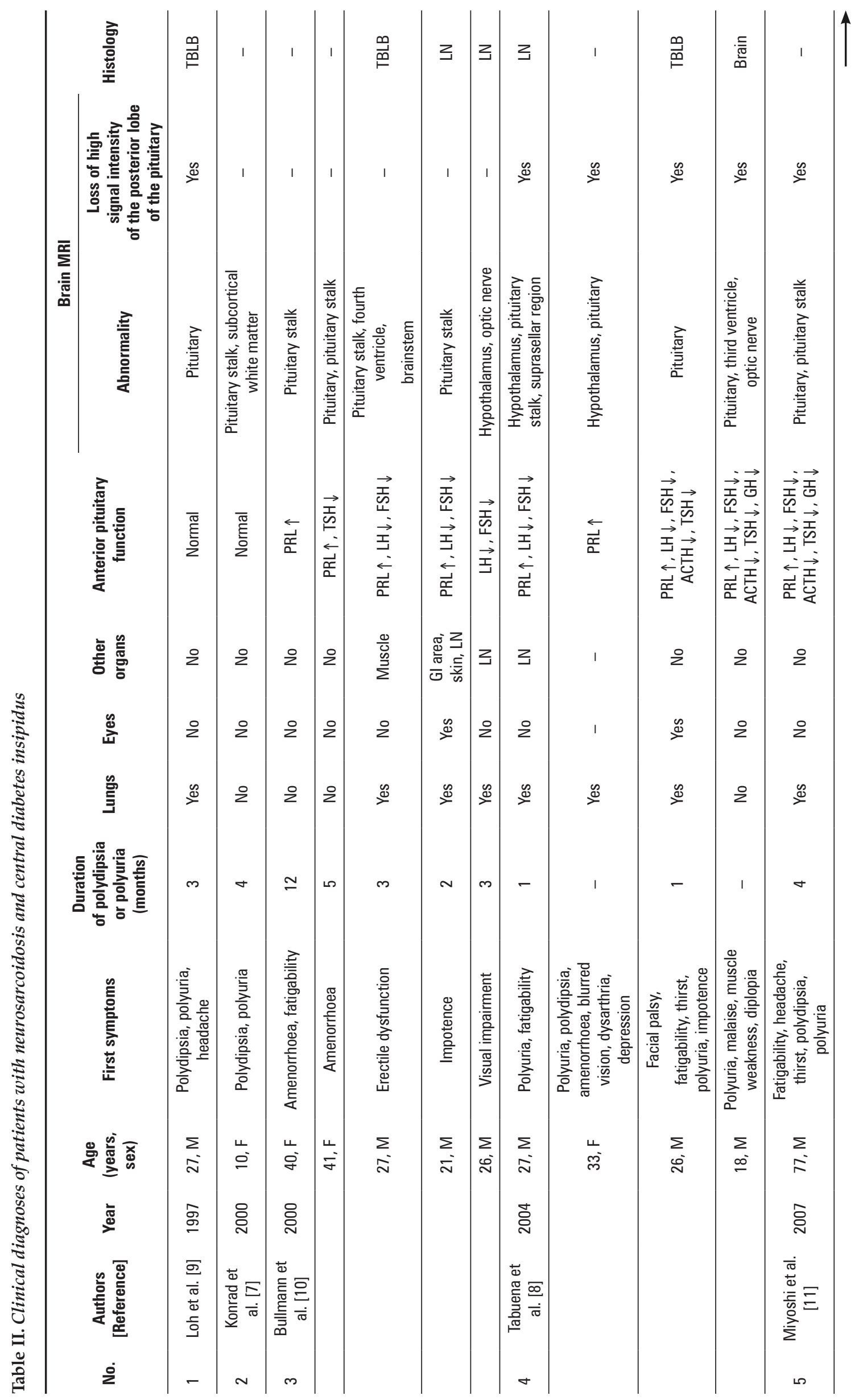




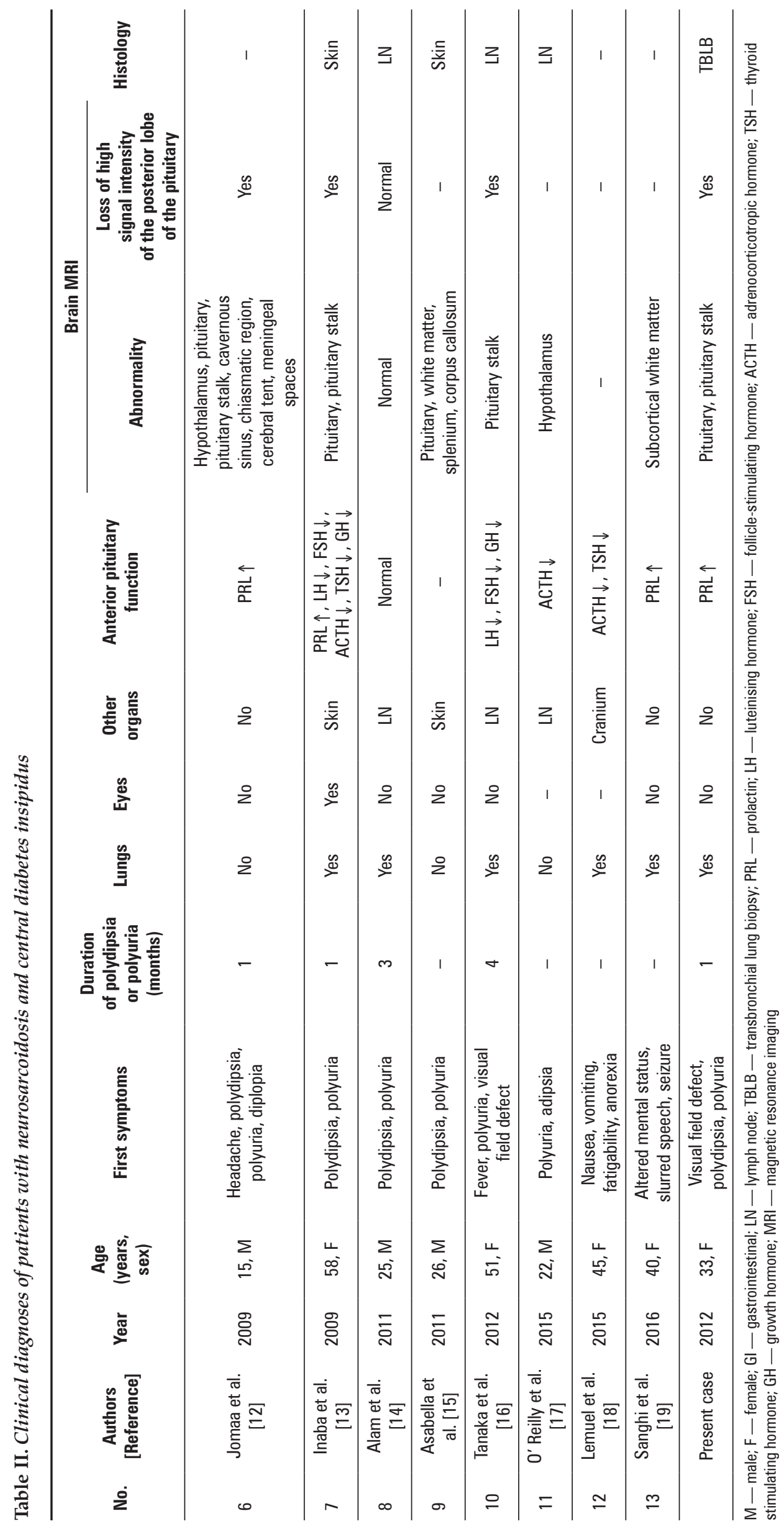




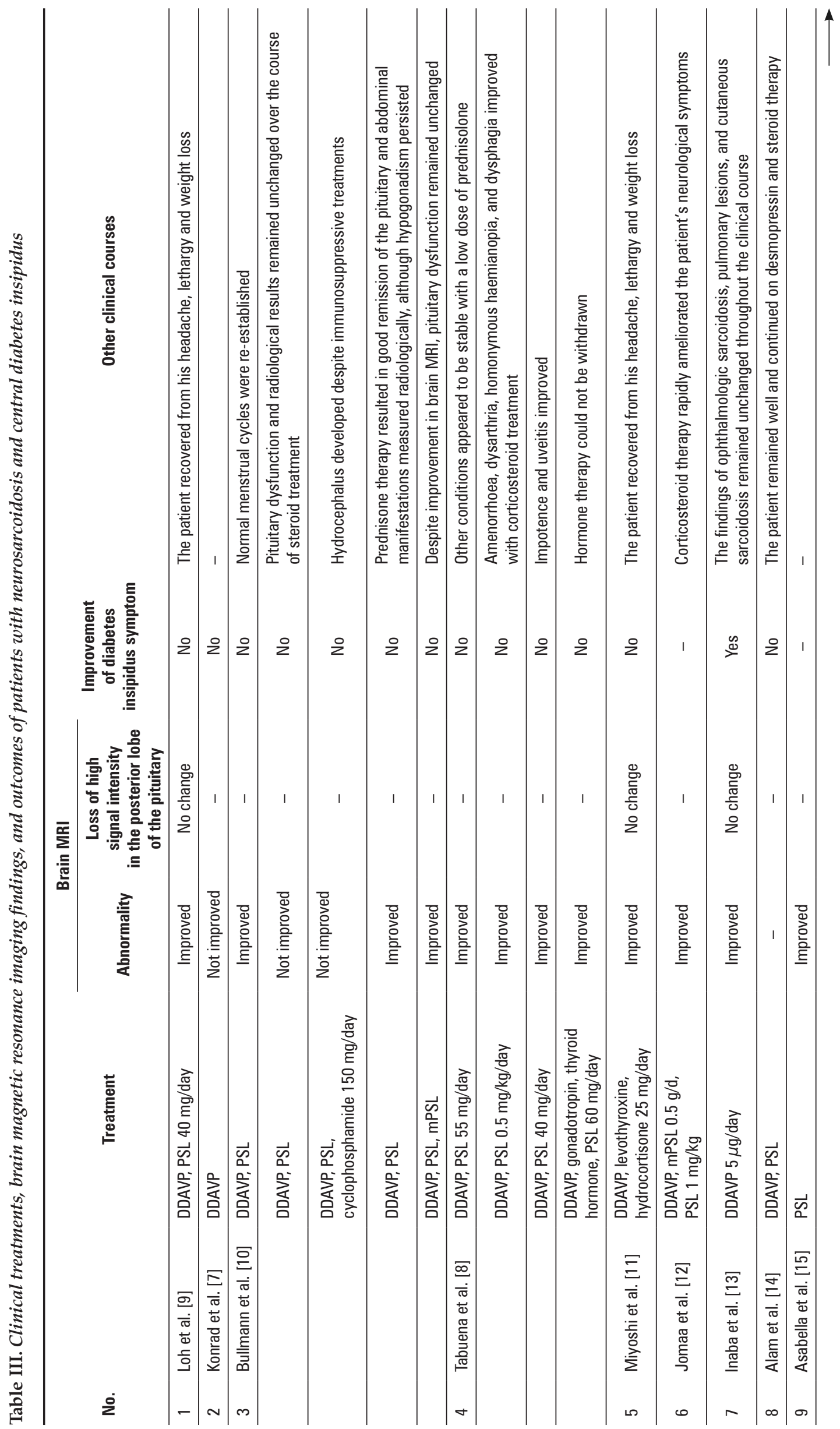


included a follow-up period that was long enough to demonstrate improvements in neurosarcoidosis with CDI.

\section{Conclusion}

During the clinical course of neurosarcoidosis and CDI, symptoms frequently do not improve even when improvements are observed in the brain MRI findings following treatment, which highlights the need for vigilance in clinical practice when assessing the progress of neurosarcoidosis and CDI. More studies with detailed pathological analyses and longer follow-up periods are necessary.

\section{Founding and disclosure}

No sources of funding were used to assist in the preparation of this manuscript. No conflicts of interest are directly relevant to the content of this manuscript.

\section{References}

1. Heinle R, Chang C. Diagnostic criteria for sarcoidosis. Autoimmun Rev. 2014; 13(4-5): 383-387, doi: 10.1016/j.autrev.2014.01.035, indexed in Pubmed: 24424172.

2. Bihan H, Christozova V, Dumas JL, et al. Sarcoidosis: clinical, hormonal, and magnetic resonance imaging (MRI) manifestations of hypothalamic-pituitary disease in 9 patients and review of the literature. Medicine (Baltimore). 2007; 86(5): 259-268, doi: 10.1097/MD.0b013e31815585aa, indexed in Pubmed: 17873755.

3. Hoitsma E, Drent M, Sharma OmP. A pragmatic approach to diagnosing and treating neurosarcoidosis in the 21st century. Curr Opin Pulm Med. 2010; 16(5): 472-479, doi: 10.1097/MCP.0b013e32833c86df, indexed in Pubmed: 20671516.

4. Langrand C, Bihan $\mathrm{H}$, Raverot $\mathrm{G}$, et al. Hypothalamo-pituitary sarcoidosis: a multicenter study of 24 patients. QJM. 2012; 105(10): 981-995, doi: 10.1093/qjmed/hcs121, indexed in Pubmed: 22753675.

5. Leonhard SE, Fritz D, Eftimov F, et al. Neurosarcoidosis in a Tertiary Referral Center: A Cross-Sectional Cohort Study. Medicine (Baltimore). 2016; 95(14): e3277, doi: 10.1097/MD.0000000000003277, indexed in Pubmed: 27057889 .

6. Terushkin V, Stern BJ, Judson MA, et al. Neurosarcoidosis: presentations and management. Neurologist. 2010; 16(1): 2-15, doi: 10.1097/NRL.0b013e3181c92a72, indexed in Pubmed: 20065791.

7. Konrad D, Gartenmann M, Martin E, et al. Central diabetes insipidus as the first manifestation of neurosarcoidosis in a 10-year-old girl. Horm Res. 2000; 54(2): 98-100, doi: 10.1159/000053239, indexed in Pubmed: 11251374 .

8. Tabuena RP, Nagai S, Handa T, et al. Diabetes insipidus from neurosarcoidosis: long-term follow-up for more than eight years. Intern Med. 2004; 43(10): 960-966, doi: 10.2169/internalmedicine.43.960, indexed in Pubmed: 15575248.

9. Loh KC, Green A, Dillon WP, et al. Diabetes insipidus from sarcoidosis confined to the posterior pituitary. Eur J Endocrinol. 1997; 137(5): 514-519, indexed in Pubmed: 9405032.

10. Bullmann C, Faust M, Hoffmann A, et al. Five cases with central diabetes insipidus and hypogonadism as first presentation of neurosarcoidosis. Eur J Endocrinol. 2000; 142(4): 365-372, indexed in Pubmed: 10754478.

11. Miyoshi T, Otsuka F, Takeda M, et al. An elderly patient with sarcoidosis manifesting panhypopituitarism with central diabetes insipidus. Endocr J. 2007; 54(3): 425-430, indexed in Pubmed: 17446653.

12. Jomaa R, Sfar MH, Mhenni SY, et al. Isolated neurosarcoidosis revealed by diabetes insipidus, visual loss and diplopia in a child patient: a diagnostic problem. Clin Pediatr Endocrinol. 2009; 18(1): 51-54, doi: 10.1297/cpe.18.51, indexed in Pubmed: 24790380.

13. Inaba H, Suzuki S, Shigematsu S, et al. Spontaneous remission of diabetes insipidus due to CNS sarcoidosis. Intern Med. 2009; 48(4): 225-229, doi: 10.2169/internalmedicine.48.1583, indexed in Pubmed: 19218773.

14. Alam T, Thomas S. Diabetes insipidus secondary to sarcoidosis presenting with caseating granuloma. BMJ Case Rep. 2011; 2011, doi: 10.1136/bcr.01.2011.3702, indexed in Pubmed: 22707619. 
15. Asabella AN Gatti P Notaristefano A, et al. F-18 FDG $\mathrm{PET} / \mathrm{CT}$ in the diagnosis of a rare case of neurosarcoidosis in a patient with diabetes insipidus. Clin Nucl Med. 2011; 36(9): 795-797, doi: 10.1097/RLU.0b013e318219b28b, indexed in Pubmed: 21825853.

16. Tanaka Ki, Yamamoto M, Okazaki K, et al. Partial improvement of anterior pituitary deficiency following steroid treatment in a patient with neurosarcoidosis accompanied by central diabetes insipidus. Intern Med. 2012; 51(16): 2175-2179, doi: 10.2169/internalmedicine.51.6957, indexed in Pubmed: 22892499.

17. O'Reilly MW, Sexton DJ, Dennedy MC, et al. Radiological remission and recovery of thirst appreciation after infliximab therapy in adipsic diabetes insipidus secondary to neurosarcoidosis. QJM. 2015; 108(8) 657-659, doi: 10.1093/qjmed/hct023, indexed in Pubmed: 23417911.

18. Non L, Brito D, Anastasopoulou C. Neurosarcoidosis-associated central diabetes insipidus masked by adrenal insufficiency. BMJ Case Rep. 2015; 2015, doi: 10.1136/bcr-2014-206390, indexed in Pubmed: 25612752
19. Sanghi V, Kapoor A. Unusual Presentation of Central Diabetes Insipidus in a Patient With Neurosarcoidosis. J Investig Med High Impact Case Rep. 2016; 4(3): 2324709616667511, doi: 10.1177/2324709616667511 indexed in Pubmed: 27652275.

20. West SG. Current management of sarcoidosis I: pulmonary, cardiac, and neurologic manifestations. Curr Opin Rheumatol. 2018; 30(3): 243-248, doi: 10.1097/BOR.0000000000000489, indexed in Pubmed: 29389828.

21. Kaliszky Z, Walker A, Tyor WR. Therapeutic options in neurosarcoidosis. Expert Rev Neurother. 2002; 2(5): 703-708, doi: 10.1586/14737175.2.5.703, indexed in Pubmed: 19810986.

22. Sugiyama K, Mukae H, Sakamoto N, et al. [A case of sarcoidosis with diabetes insipidus]. Nihon Kokyuki Gakkai Zasshi. 2007; 45(1): 105-109, indexed in Pubmed: 17313038.

23. Johnston PC, Chew LS, Hamrahian AH, et al. Lymphocytic infundibulo-neurohypophysitis: a clinical overview. Endocrine. 2015; 50(3): 531-536, doi: 10.1007/s12020-015-0707-6, indexed in Pubmed: 26219407 